Revista Ingeniería y Región. 2015;14(2): 55-63

\title{
Monitoreo remoto a sistemas de riego por bombeo eléctrico a cultivos de arroz en el departamento del Huila
}

\section{Remote monitoring of irrigation systems for pumping electric to farming of rice in the department of Huila}

\author{
Jesús David Quintero P. ${ }^{1}$ Martín Diomedes Bravo Obando ${ }^{2}$ y Albeiro Yaime Ramírez ${ }^{3}$
}

\begin{abstract}
Resumen
Se ha desarrollado un sistema integral que se encarga de monitorear el estado de las principales variables de un sistema de riego (caudal de agua, temperatura y estado magnético del motor de bombeo) y enviar esta información en tiempo real al usuario final mediante Internet a través de una página web que se diseñó para este fin. El sistema está compuesto por tres sensores (temperatura, caudal y estado magnético), los cuales se conectan a una tarjeta electrónica de desarrollo (Arduino) encargada de la adquisición y procesamiento de la magnitud de las variables, dos módulos de transmisión inalámbrica (XBee) que intercomunican la tarjeta electrónica con un computador (servidor web), el cual con una conexión a Internet se encarga de enviar ésta información al usuario o administrador del sistema por E-mail. El sistema de monitoreo está planteado para que Electrohuila S.A E.S.P pueda ofrecerlo como servicio de valor agregado a usuarios agrícolas con sistemas de riego con bombeo eléctrico. Electrohuila S.A E.S.P es la empresa encargada del servicio de distribución de energía eléctrica en el departamento del Huila, Colombia.
\end{abstract}

Palabras clave: Monitoreo, Variables, XBee, Arduino, Software Libre, Servidor Web.

\begin{abstract}
It was developed an integrated system that is responsible for monitoring the state of the main variables of an irrigation system (water flow, temperature and magnetic state of the pump motor) and send this information to the end user in real time via Internet through a website that was designed for this purpose. The system comprises three sensors (temperature, flow and magnetic state), which a smartcard development (Arduino) responsible for the acquisition and processing of the magnitude of the variable, two modules wireless transmission (XBee) connect which connect the electronic board to a computer (web server), which with an Internet connection is responsible for sending this information to the user or system administrator by email. The monitoring system is propose to Electrohuila SA ESP may offer it as a value-added service users to agricultural irrigation systems with electrical pumping. Electrohuila ESP SA is the service company responsible for distribution of electricity in the department of Huila, Colombia.
\end{abstract}

Key words: Monitoring, Variable, XBee, Arduino, Free Software, Web Server.

1 M.sC. en Telecomunicaciones. Docente Universidad Surcolombiana Neiva. Avenida Pastrana Carrera 1 ${ }^{\text {a }}$. jdavid@ usco.edu.co

$2 \operatorname{Dr}(\mathrm{c})$ en Teleinformatica. Docente Universidad Surcolombiana Neiva. Avenida Pastrana Carrera 1 ${ }^{\mathrm{a}}$. martin.bravo @ usco.edu.co 3 Ingeniero Electrónico. Universidad Surcolombiana Neiva. Avenida Pastrana Carrera 1a. u2009179069@usco.edu.co1

Recibido: 30 abril 2015 Aceptado: 5 Agosto 2015 


\section{Introducción}

Hoy en día la electrónica se encuentra apoyando todos los campos del conocimiento, entre los que se encuentra el agro. El avance tecnológico ha permitido optimizar el aprovechamiento de los recursos naturales y aumentar la producción agrícola. El presente proyecto se fundamenta en una aplicación tecnológica que permite el monitoreo de un sistema industrial, el cual toma mediciones remotas de magnitudes físicas que son transmitidas en tiempo real a un sistema de cómputo el cual registra, procesa y tabula la información obtenida para ser enviada al usuario, en este caso, el supervisor de un sistema de riego para un cultivo de arroz. El sistema diseñado permite enviar las variables a un servidor mediante el acceso a un proveedor de servicios de internet "ISP".

Un sistema de riego por bombeo eléctrico es una estructura que permite la aplicación de agua por métodos artificiales a cualquier superficie dedicada a un determinado cultivo de plantas, el cual consta de una serie de componentes que establecen ciertos tipos de sistemas de riego como por ejemplo, aspersión, goteo, subterráneo, inundación, entre otros. En el caso de arroz se utiliza el método de inundación debido a que los suelos inundados ofrecen un ambiente único para el crecimiento y nutrición del arroz (Infoagro, 2015).

El servidor que se utiliza para este aplicativo, usa una base de datos, la cual almacena los registros de las variables caudal de agua, temperatura y estado magnético del motor empleado para el bombeo del agua, monitoreando el comportamiento del sistema continuamente, a través de un módulo de adquisición y transmisión de datos llamado Arduino.

La supervisión de un sistema de bombeo eléctrico en cultivos de arroz tiene bastante dificultad y es engorroso para un productor agrícola, debido a la necesidad de estar en todo momento en las instalaciones y dificultad para comunicarse con quien suministra la energía eléctrica, además de las fallas que se puedan presentar en el sistema de bombeo por la no atención en las variables a monitorear. En un cultivo de arroz es imprescindible el suministro de agua continuo para poder mojar grandes extensiones de tierra, el no hacerlo implica tener que volver a iniciar la moja y perder tiempo y dinero en el reinicio del proceso. Es por ello que la utilización de este diseño electrónico se convierte en una herramienta sostenible y eficiente para facilitar el monitoreo de un sistema de bombeo eléctrico y evitar inconvenientes posteriores.
Estos sistemas tecnológicos que anteriormente representaban un alto costo para el agricultor, han tomado fuerza debido a que la tecnología cada vez está más al alcance económico de algún pequeño o mediano productor, en donde la tecnificación de los sistemas de bombeo es una gran herramienta para la producción y mejoramiento de la calidad de los productos finales. La empresa Electrohuila pretende llevarlo al productor agrícola que utilicen bombeos eléctricos, logrando el mejoramiento las condiciones del sistema de bombeo eléctrico del usuario rural y contribuyendo con el desarrollo de la región huilense.

\section{Metodología}

El sistema que se encuentra modelado en la Figura 1, está compuesto por tres sensores que se encuentran conectados por cable a el Arduino Mega, un módulo XBee como un dispositivo de salida hasta otro módulo XBee que se encuentra conectado al servidor en el cual se procesa la información y es transmitida vía web al usuario final.

Con el desarrollo del proyecto se construye una innovadora estrategia de supervisión y monitoreo de sistemas de riego por bombeo eléctrico, el cual tiene como objetivo garantizar y contribuir con el correcto desarrollo del cultivo y ayudar a la protección del motor, facilitando la supervisión de su adecuado funcionamiento, además permitiendo el monitoreo desde cualquier lugar a través de internet.

\subsection{Hardware del sistema de monitoreo remoto}

El sistema de monitoreo remoto está integrado por una tarjeta de desarrollo Arduino Mega 2560, un sensor de temperatura, un sensor de caudal de agua, un sensor de estado magnético, dos módulos de comunicación XBee S1 y un computador utilizado como servidor web.

\subsubsection{Arduino Mega 2560}

ElArduino Mega 2560 es una placa electrónica basada en el ATmega2560. Tiene 54 entradas / salidas digitales (de las cuales 15 pueden utilizarse para salidas PWM), y 16 entradas analógicas. Esta tarjeta lee los puertos digitales de entrada en donde se conectan los sensores para calcular y muestrear la magnitud de las variables (Temperatura, caudal y estado magnético), y enviar al puerto serial de salida un paquete con el muestreo de dichas señales, junto a una bandera de referencia (' $x$ ') que separa dichos paquetes (Arduino, 2015). 


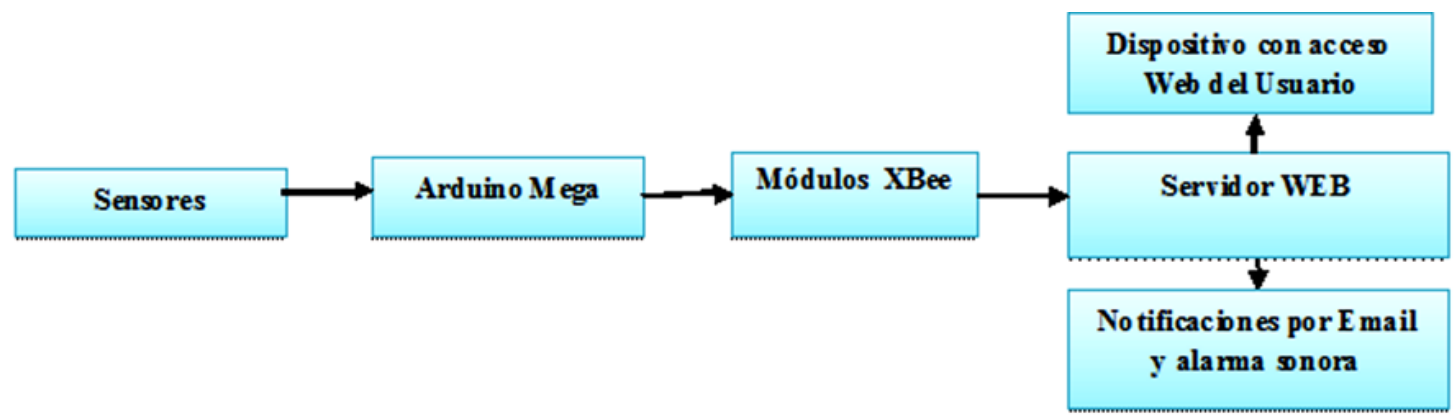

Figura 1. Esquema general del proyecto Monitoreo Remoto a Sistemas de Riego.

\subsubsection{TEM01072B, Sensor de caudal}

Encargado de medir el flujo de agua en el sistema de riego. Cuando este se encuentra por fuera del rango permitido según la programación del riego, el sistema envía mensajes de alerta al usuario notificando la falla presentada para que pueda ser corregido en el menor tiempo posible y así proteger el cultivo de los puntos críticos.

\subsubsection{DFR0024, Sensor de temperatura}

Es un dispositivo capaz de interpretar señales de cambio de temperatura en el motor y así ayudar a prevenir averías en el mismo. El sensor es instalado junto al armazón del motor y ayuda a detectar sobrecargas de consumo de corriente que elevan su temperatura, debido a fricción en los ejes y obstrucción en las bandas de transmisión (DFRobot, 2015).

\subsubsection{DFR0033, Sensor Magnético}

Este sensor es usado para detección magnética, tiene un rango de detección de hasta $3 \mathrm{~cm}$ de distancia. El sensor es instalado cerca a las bobinas del motor, en donde detecta el campo magnético de éstas para indicar el estado funcional del motor (Encendido o Apagado). Con esto se detectan posibles fallas en el circuito de suministro de energía eléctrica del motor y se advierte la necesidad de poner en marcha el sistema de riego de respaldo basado en motor a combustión, mientras se repare el suministro eléctrico (DFRobot, 2015).

\subsubsection{XBEE S1}

Es un dispositivo de comunicación inalámbrica compatible con el estándar de comunicaciones IEEE 802.15.4 o ZigBee, opera con un voltaje de alimentación de $3.3 \mathrm{~V}$, velocidad de transmisión de hasta 250 Kbps y un alcance de 100 metros en línea libre de visión(M.U., 2015).

\subsubsection{Computador, Servidor web}

Es el dispositivo al que llega la información procedente del Arduino, se encuentra conectado a Internet para llevar la información sobre el estado del sistema de riego hasta el usuario final a través de la página web que se diseñó. Además, ante cualquier estado crítico del sistema genera notificaciones que son enviadas al correo electrónico del usuario y ejecuta sonidos de alarma en el dispositivo (PC, Smartphone, etc.) en donde se esté monitoreando el sistema de riego desde internet.

\subsection{Software del sistema de monitoreo remoto}

El código de Arduino Mega 2560 se desarrolló en Arduino IDE 1.0.5. El programa que ejecuta Arduino Mega 2560 consiste en leer los puertos de los sensores, calcular el valor de los sensores debido a que estos son digitales, y enviarlos al puerto serial en donde se encuentra instalado un módulo de transmisión inalámbrica XBEE serie 1.

El otro módulo XBEE serie 1 se encuentra conectado al servidor en un puerto serial COM, y se encarga de recibir los paquetes que contienen la información de la magnitud de las variables del sistema de riego.

\subsubsection{Aplicación de almacenamiento}

La aplicación de almacenamiento desarrollada en Lazarus Free Pascal (Figura 2), cumple la función de mostrar el almacenamiento de las variables que van llegando desde la tarjeta Arduino Mega 2560. La estructura del programa se basa en tres funciones principales: Abrir el puerto serial COM del servidor y leer los paquetes con los datos de magnitud de las variables. Filtrar los paquetes erróneos. Guardar la magnitud de las variables sobre la base de datos y mostrar la visualización local en tiempo real. 


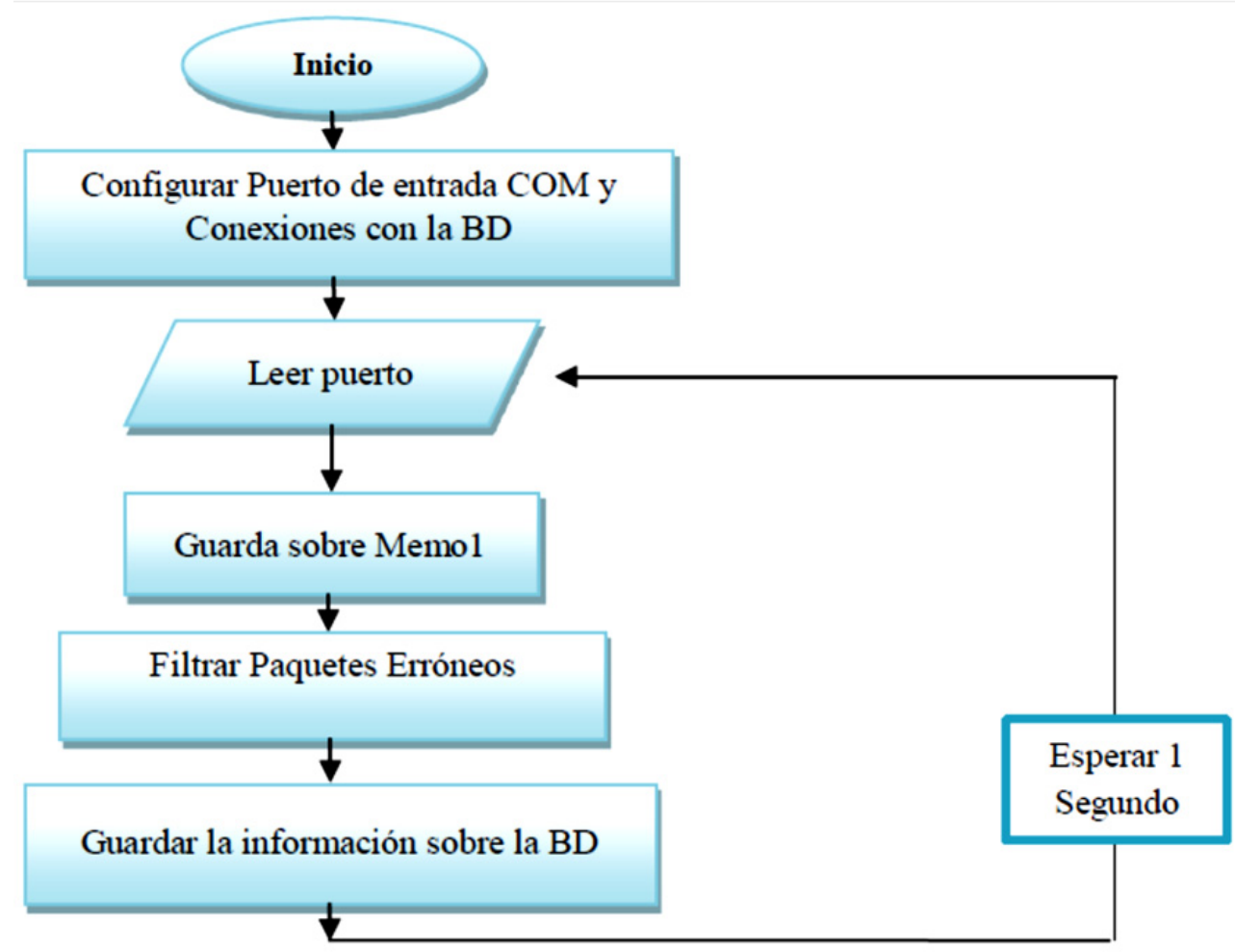

Figura 2. Estructura de la aplicación de almacenamiento

\subsubsection{Servidor web Apache}

Este software libre es utilizado para instalar el servidor WEB en el ordenador y de esta forma publicar la página web a través de internet, utilizando únicamente la dirección IP de este ordenador. Además es necesario habilitar el puerto 80 en el Cortafuegos del sistema operativo. La página y todos sus archivos se deben ubicar en la carpeta "www" del servidor web, el archivo de la página de inicio (index.php) será el primero en ejecutarse al entrar a la dirección IP del servidor mediante un navegador web.

\subsubsection{Servidor DNS Gratuito}

Utilizado para traducir la dirección IP del ordenador donde está instalado el servidor WEB a un nombre de página Web determinado, y de esta forma facilitar el acceso. Actualmente existe una gran cantidad de servidores DNS (Domain Name System), no obstante, el servidor gratuito utilizado en este proyecto es "No-Ip" el cual es uno de los más utilizados a nivel mundial.

\subsubsection{DUC (Dynamic Update Client)}

El servidor DNS gratuito "No-Ip", permite a sus usuarios descargar la aplicación DUC (Figura 3), la cual se encarga de actualizar la IP del servidor web ante el servidor DNS. Por ser una IP dinámica, la dirección IP del servidor puede cambiar ocasionalmente. Sin embargo, gracias a esta aplicación, se puede hacer uso del mismo nombre (monitoriego.noip.me) sin ningún inconveniente (NOIP, 2015).

\section{Resultados}

\subsection{Aplicación de visualización}

La aplicación de almacenamiento emplea indicadores para mostrar la magnitud de las variables del sistema de riego (Figura 4), estos indicadores muestran la información del caudal en Litros/Segundo, la temperatura en grados centígrados, el estado magnético del motor On/Off 1. Esta aplicación se conecta con una base de datos para guardar la información de estas variables.

\subsection{Página Web}

Se construye una página web compuesta a su vez por una sub-páginas, una página de inicio (Figura 5) para seguridad y una página principal de monitoreo a la que solo tiene acceso el usuario final desde cualquier lugar del mundo mediante internet. 


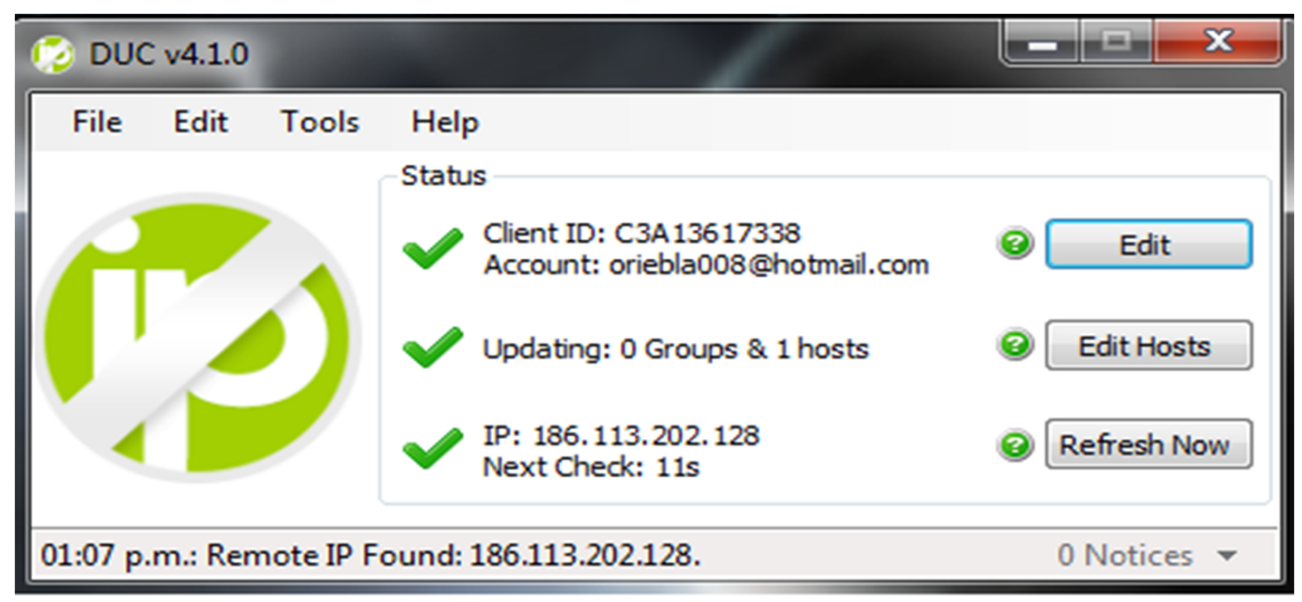

Figura 3. Actualizador de IP dinámico.
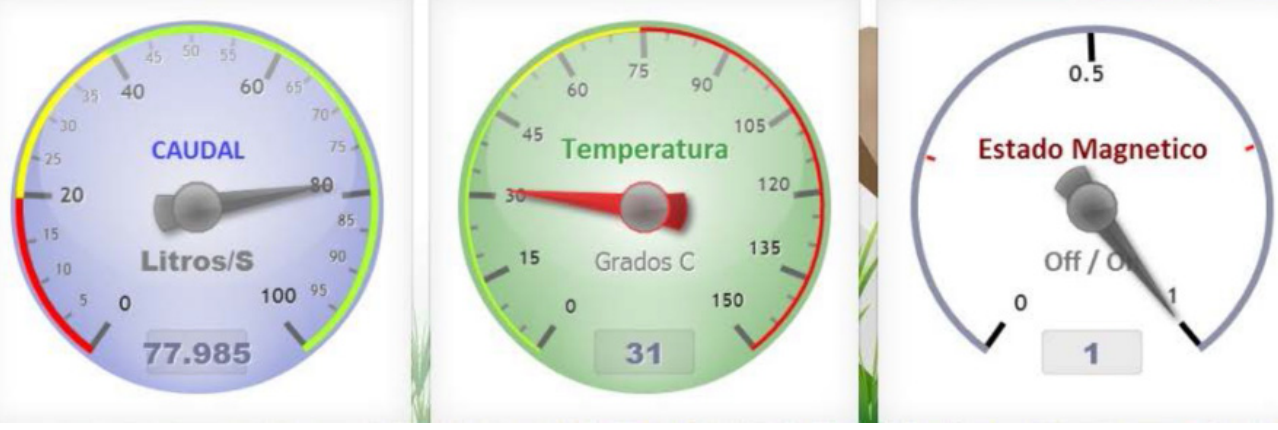

Figura 4. Aplicación de almacenamiento y visualización local

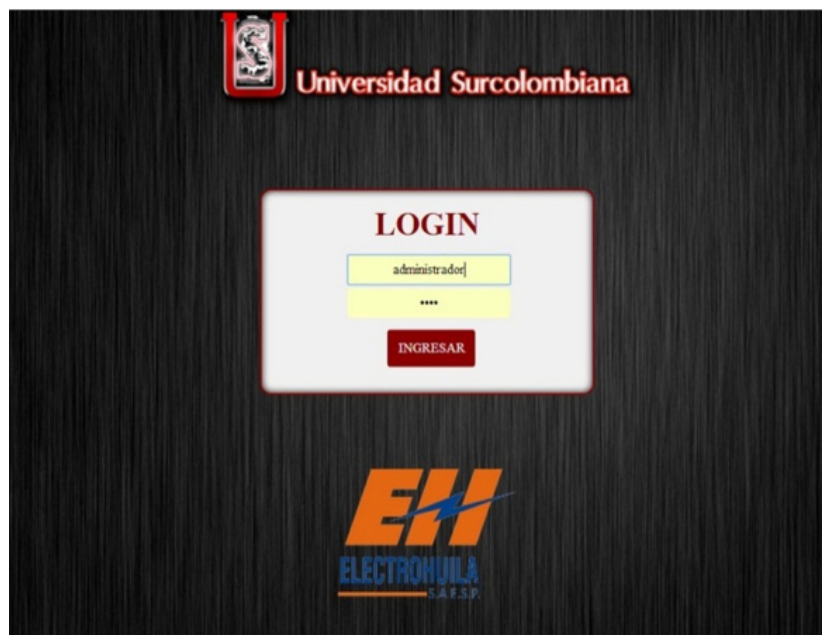

Figura 5. Página web de inicio.

La página web de inicio permite el acceso solamente al usuario final mediante el uso de un nombre de usuario y contraseña determinada por él. En esta página se captura mediante método \$_POST lo que se va escribiendo en los campos y luego de presionar el Botón "INGRESAR", se ejecuta un código PHP que conecta con la base de datos y extrae la información de usuarios, para compararlos con los valores ingresados en los campos, y permitir o denegar el acceso a la página principal.

La página principal cumple la función de visualización a través de unos indicadores llamados "calibradores" (gauges) cuyo código fue prediseñado en Codeproject. La auto-actualización de los visualizadores se ejecuta a través de una rutina que se ejecuta cada 1 segundo (Thirugnanam, 2015).

Además de visualizar, esta página principal es la encargada de actualizar en tiempo real las variables que se encuentran monitoreado, cuando ocurra un evento pueda enviar notificaciones al correo electrónico con la información del estado del sistema de riego y la hora exacta del evento. También en la misma rutina de revisión, se activa un sonido de alarma en cualquiera de las variables dependiendo de los valores que se hayan considerado críticos, para este caso si el motor se encuentra con una temperatura superior a 75 grados, el caudal por debajo de los 20 litros/segundo o el estado magnético es cero se consideran alarmas críticas para el usuario. 
Las unidades correspondientes son: caudal en litros por segundo y temperatura en grados centígrados. El estado magnético es adimensional debido a que este solamente representa el funcionamiento del motor (encendido o apagado).

\subsubsection{Notificaciones por Correo Electrónico}

e elaboró dentro de la página web, un sistema que genera correos electrónicos (Figura 6) para notificar al usuario de cualquier estado crítico o sea una variable que este por fuera del rango programado en su sistema de riego. El intervalo en que se envían los correos electrónicos es determinado previamente por el usuario (Ustarroz, 2015).

\subsubsection{Alarmas de sonido}

El sistema de alarmas dentro de la página web se encarga de revisar las variables y determinar si alguna de estas se encuentra fuera del rango programado, para activar una alarma de sonido en el dispositivo donde el usuario final este monitoreando su sistema de riego. El sonido proviene de la activación de un archivo de sonido llamado alarm. WAV, el cual se encuentra ubicado dentro de la carpeta " $w w w$ " del servidor web.

\subsection{Servicio de valor agregado}

El presente proyecto se planteó como servicio de valor agregado para usuarios de Electrohuila, los cuales podrán acceder fácilmente a este servicio y pagarlo en la factura de energía. Como único requisito el usuario debe tener un computador ya sea portátil o de mesa con acceso a internet en donde será instalado el servidor web, teniendo en cuenta que la distancia máxima entre la tarjeta Arduino Mega 2560 y el servidor web debe ser de 100 metros debido al alcance de los módulos de transmisión inalámbricos XBee. No es comparable con otros sistemas ya que no se tienen implementado por empresa alguna en la actualidad.

\subsubsection{Beneficio del proyecto}

Los beneficios para Electrohuila equivalen directamente a las utilidades, debido a que el servicio de monitoreo no incluye precio de los activos con el fin de reducir el costo del servicio al usuario y permitir a Electrohuila ser propietario único de los activos.

Los beneficios para el Usuario son todos los relacionados con la eficacia y apoyo de este Sistema de Monitoreo remoto para aumentar la eficiencia energética del Sistema de Riego, evitar daños por elevación de temperatura en el motor eléctrico, prevenir deterioros en el cultivo por faltas de agua y mejorar el aprovechamiento del recurso hídrico. El proyecto piloto se instaló en la finca la Rivera en la ciudad de Neiva y ha tenido gran acogida por los propietarios. En la figura 7 se muestra el prototipo mostrado y en la figura 8 instalado.

\subsubsection{Calculo del Costo del Servicio para el cliente}

Para calcular el costo que debe asumir el usuario se debe tener en cuenta el total de la inversión, la vida útil de los activos, el tiempo en que se desea recuperar la inversión y tiempo de suministro del servicio mensual (Trabajo MX, 2015).

La vida útil del Sistema de Monitoreo basado en la placa Arduino, está alrededor de 10.000 .000 operaciones y a las condiciones del lugar donde esté instalado. En este caso la placa Arduino Mega 2560 no será expuesta a altas temperaturas ni vibraciones extremas, además reposa 8 horas diarias, por lo tanto la vida útil se extiende más allá de 5 años (Young, 2015).

Vida útil: 5 años (mínimo en condiciones normales de trabajo), Tiempo de recuperación de inversión: 12 meses.

El costo del servicio para el Usuario que cumple con el único requisito es el mostrado en la Tabla 1.

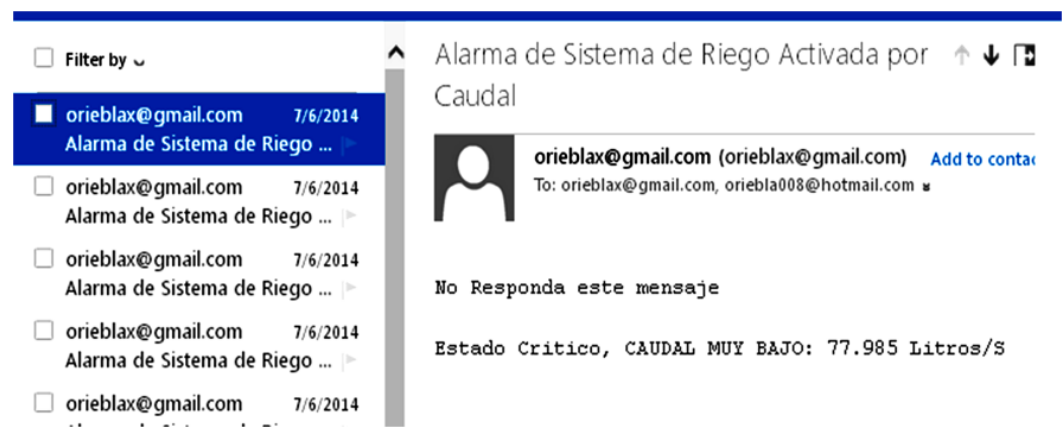

Figura 6. Notificaciones en el correo electrónico. 


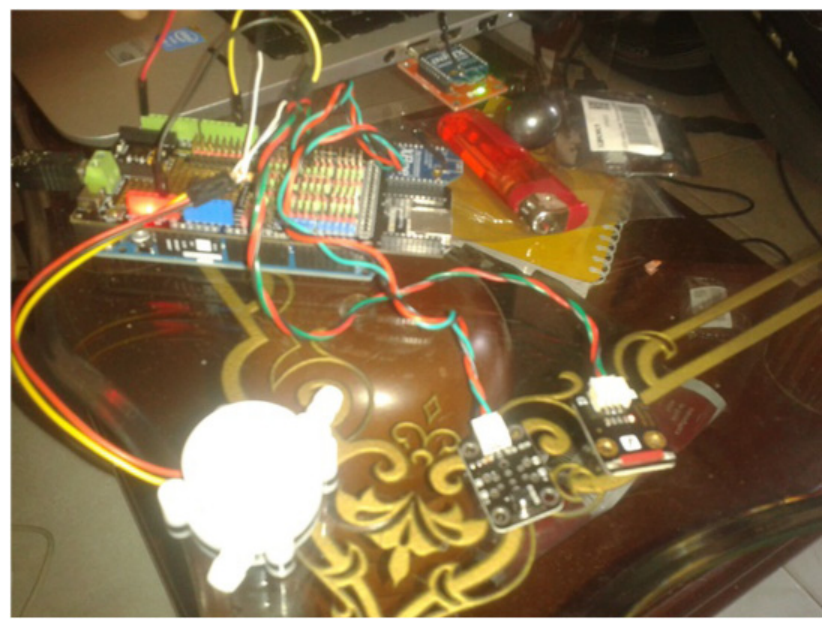

Figura 7. Prototipo finalizado.

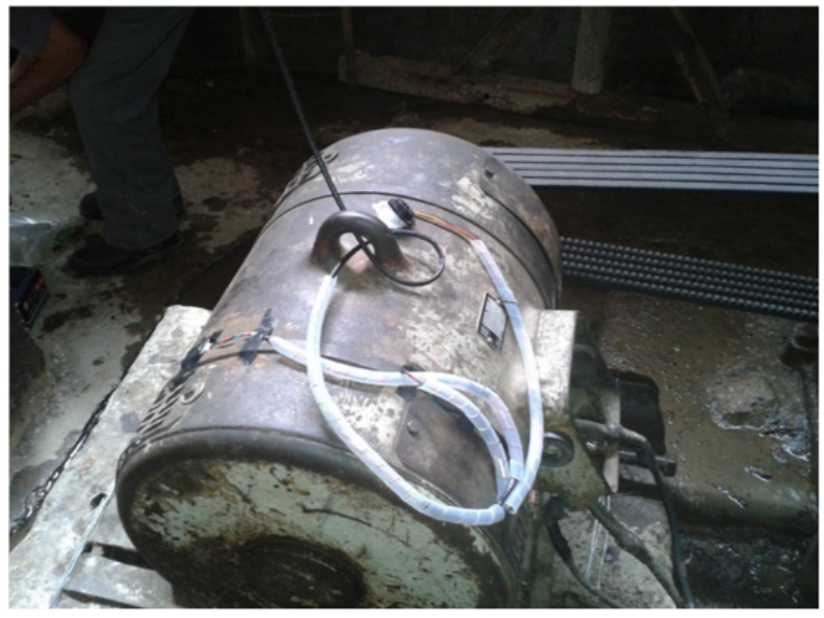

Figura 8. Prototipo montado sobre el motor eléctrico.

Tabla 1. Costo del Servicio para Usuario con Internet Instalado.

\begin{tabular}{l|cc}
\hline Total Inversión Electrohuila & $\$ 611.308$ \\
\hline Gastos Operacionales: No requiere operarios & $\$$ & - \\
\hline Recuperación Mensual de inversión (Inversión/5 años/12 meses) & $\$$ & 10.188 \\
\hline Internet mensual & $\$$ & - \\
\hline Utilidades: 4\% Sobre inversión Total & $\$$ & 24.452 \\
\hline Costo Total Servicio para el Usuario & $\$$ & $\mathbf{3 4 . 6 4 0}$ \\
\hline Valor Servicio Electrohuila & $\mathbf{\$ 3 4 . 6 4 0}$ \\
\hline IPC anual (Junio de 2015) & 0,0279 \\
\hline Incremento Anual Servicio: Incremento en Utilidad mes & $\$$ & 70 \\
\hline
\end{tabular}

El valor mensual facturado por concepto de servicios de valor agregado bajo estos parámetros sería de \$34.640 más los impuestos que estipula el Estado, como el IVA.

\section{Conclusiones}

El Sistema de Monitoreo Remoto desarrollado mediante tecnología Arduino le ofrece al usuario información en tiempo real, completa, detallada y a distancia; el usuario también podrá recibir notificaciones sobre cualquier eventualidad en el sistema de riego para evitar poner en riesgo el cultivo.

El Sistema de Monitoreo Remoto puede adaptarse fácilmente para supervisar muchos otros sistemas e incluso si se implementan actuadores se puede obtener automatización de diferentes procesos industriales, esto se debe a la versatilidad de Arduino y a que se ha desarrollado una aplicación y una página web muy flexibles.
El Sistema de Monitoreo Remoto se realizó mediante software libre para reducir costos en su desarrollo, haciéndolo muy eficiente en la prevención de insuficiencias en el cultivo, mejorando el aprovechamiento del recurso hídrico, y a la vez siendo rentable para el usuario pues no tiene que pagar licencias ni prestaciones por el servicio.

La tarjeta de desarrollo Arduino se programa con software libre, es amigable al programador por sus muchas librerías que se modifican de acuerdo con los requerimientos y se adecúa para diferentes funciones como adquisición de señales de sensores y transmisión de datos de forma inalámbrica usando conjuntamente módulos externos para este fin.

Con la implementación del proyecto Monitoreo Remoto a Sistemas de Riego, Electrohuila contribuirá con el desarrollo tecnológico del departamento del Huila, y a su vez generar utilidades en servicios de valor agregado. 
Se recomienda monitorear más variables, ya que este sistema cuenta con todo lo necesario para poder extender su uso hacia otros sensores, y con esto construir un sistema de monitoreo más complejo de acuerdo a las necesidades del usuario.

Se recomienda la utilización de nuevas versiones de Arduino y XBee que lleguen al mercado, así como el acople de nuevas variables que requiera el cliente.

Se recomienda realizar un estudio de las pérdidas de paquetes en los enlaces.

Se recomienda realizar un estudio costo beneficio a los cultivos que tienen esta aplicación con los que no la utilizan.

\section{Referencias bibliográficas}

Maloney T., 2006. Electrónica Industrial Moderna, PEARSON EDUCACIÓN. 221 pp.

Muños R., 2009. Mantenimiento de Portales de Información, VISIÓN LIBROS, Madrid. 223 pp.

Sobrevilla M, A., 2008. Sensores Eléctricos, Aplicables en informática, mediciones, regulación y control automático. ALSINA, Buenos Aires. 192 pp.

Agromeat - Sistema de riego inteligente. Buenos Aires, Argentina. Consultado el 15 de junio de 2015. http:/ /www.agromeat.com/111203/desarrollan-un-sistema-de-riego-inteligente-que-sepuede-monitoreardesde-internet

Sistemas de riego, Definición de riego Consultado el 10 de junio de 2015. http://es.slideshare.net/csemidei/ sistemas-de-riegopresentation

El cultivo del arroz, adaptación del arroz a los suelos inundados Consultado el 10 de junio de 2015. http:/ /www.infoagro.com/herbaceos/cereales/arroz.htm

Grupo IRC -Innova Technologies. Zaragoza, España. Consultado el 10 de junio de 2015. http: //www.radio comunicaciones.net/telemetria.html

Diseño de páginas web HTML5, Diseño web adaptado a dispositivos móviles. Consultado el 10 de junio de 2015 http://www.ipixelestudio.com/blog/disenoweb-adaptado-dispositivos-movilesrespon sivedesign.html
ARDUINO, Plataforma de Código Abierto. Consultado el 10 de junio de 2015. http://www. arduino.cc/es/.

Secretaria de Agricultura y ganadería - Manual Técnico para el cultivo del arroz (Oryza Sativa). Comayagua, Honduras. Consultado el 10 de junio de 2015. http:/ /curlacavunah.files.wordpress.com/2010/04/el-cultivo-delarroz.pdf

Tutorial sensor de flujo de Agua. Seeedstudio. Consultado el 12 de junio de 2015. http://www. see edstudio.com/forum/viewtopic.php? $\mathrm{f}=4 \& \mathrm{t}=$ $989 \& p=3632$

18B20 Sensor de Temperatura, DFRobot Wiki. Consultado el 10 de junio de 2015 http://www. dfrobot. com/wiki/index.php/18B20_Temerature_Sensor (Arduino_Compatible)_(SKU:_DFR0024)

Sensor magnético digital compatible con Arduino (DFR0033), DFRobot Wiki. Consultado el 15 de junio de 2015. http://www.dfrobot.com/wiki/ index.php/Digital_magnetic_sensor_(SKU: DFR0033)

ARDUINO, Plataforma de Código Abierto. Consultado el 15 de junio de 2015. http://www.arduino.cc/

ARDUINO, Plataforma de Código Abierto. Consultado el 15 de junio de 2015. http://arduino.cc/en/Main/ arduinoBoardMega2560.

ARDUINO, Plataforma de Código Abierto. Arduino Board Mega 2560. Consultado el 10 de junio de 2015. http:/ /arduino.cc/en/Main/arduinoBoard Mega2560.

Módulo Xbee 2.4 GHz Serie 1, Mecatrónica Uruguay. Consultado el 15 de junio de 2015. http://www. mecatronica.com.uy/rfwireless/93-modulo-xbee1mw-24ghz-antena-incluida-serie-1.html

Free Pascal, Descripción de Lazarus y sus aplicaciones Consultado el 10 de junio de 2015. http://wiki. freepascal.org/Overview_of_Free_Pascal_ and_Lazarus/es

Bases de datos MySQL, Dimensiones máximas de las tablas MySQL. Consultado el 10 de junio de 2015. http://dev.mysql.com/doc/refman/5.0/es/tablesize.html

HTML5, Introducción a HTML5. Consultado el 15 de junio de 2015. http://www.w3schools.com/html/ html5_intro.asp 
JavaScript, introducción a JavaScript Consultado el 25 de junio de 2015. http://librosweb.es/javascript/ capitulo_1.html

Aqua gauges usando Canvas HTML5, Proyectos de Código. Consultado el 25 de junio de 2015. http:// www.codeproject.com/Articles/304874/HTMLCanvas-Aqua-Gauge

Fundación de Software Apache, Servidor Apache. Consultado el 25 de junio de 2015 http://apache foundation.wikispaces.com/Apache+HTTP+Server
Profesores de Informática, Introducción al servicio DNS. Consultado el 25 de junio de 2015. http:// www.profesordeinformatica.com/servicios/dns/ introduccion

El precio de tus servicios, calculo general del costo de servicios. Consultado el 25 de junio de 2015. http:/ /www.trabajo.com.mx/el_precio_de_tus_ servicios.htm

Vida útil Arduino, Foro Electronics. Consultado el 25 de junio de $2015 \mathrm{http}: / /$ electronics.stack exchange.com/ questions/91025/arduinolifetime. 
